\title{
Ekphrasis : au lieu de décrire ce qu'ils voyaient, ils réalisèrent ce qui était décrit
}

Ana Vanessa Lucena, HEP BEJUNE, Suisse

\section{Le projet collection Junod}

La dynamique des activités artistiques, y compris les visites d'une collection muséale, fait partie de la façon de travailler sous les directives de la pédagogie développée, dans son livre $A$ image no ensino da arte (2009), par la célèbre didacticienne en arts visuels brésilienne, Ana Mae Barbosa. Cette méthodologie de l'enseignement artistique d'abord connue sous le nom de Méthodologie triangulaire, est aujourd'hui appelée Proposition triangulaire. Cette méthode est basée sur le triptyque esthétique et critique d'art, histoire de l'art et savoir-faire artistique. C'est-à-dire, respectivement, la lecture de l'image, son contexte historique et le travail pratico-technique en atelier. La combinaison de ces trois aspects a mis fin aux points de vue exclusivement synchroniques (lecture ou critique de l'image) et diachroniques (histoire et contextualisation), allant aussi contre l'idée du laisser-faire des activités artistiques en pratiques d'atelier et/ou du bricolage par thème, en vogue depuis plusieurs décennies, et inexplicablement toujours actives aujourd'hui. Le savoir-faire artistique pratiqué dans le projet ci-dessous considère les activités artistiques comme un tout. Conçues dans l'idée de ne pas brider la créativité et la spontanéité de l'élève, ces activités sont élaborées et dirigées dans le sens où la production plastique artistique est une étape d'apprentissage intégrée aux deux autres étapes que sont la confrontation et l'interprétation de l'image. Grâce à cette méthode, quand il est proposé à l'élève l'activité de créer une nouvelle œuvre à partir de photocopies d'œuvres déjà existantes ( $̇$ la manière de...), l'œuvre sera lue dans son contexte et par sa composition plastique. En fait, l'idée est de proposer une connaissance qui dépasse l'idée instinctive que l'on se fait de la construction d'un tableau en montrant la problématique du travail de conception de l'artiste. Il s'agit, grâce à cette démarche, de confirmer certaines capacités préalables de l'élève et développer l'acuité de son regard. Stimuler l'élève par une situation dans laquelle il se met dans le rôle de l'artiste face à la feuille blanche (ou autres supports), lui demander de vivre (dans le cas d'une copie) l'expérience, la situation, presque toujours difficile, de la décision d'un choix devant l'excès de modèles stockés par l'histoire des arts sans lui donner les «clés » du regard reviendrait à construire des apprentissages vides de sens. En effet, comment saisir les gestes et les intentions de l'artiste si on ne sait pas quoi regarder? Comment comprendre et parler des œuvres sans avoir un certain vocabulaire ? Pour cela il n'est pas nécessaire de transformer les 
leçons d'arts visuels en leçons d'histoire de l'art mais il s'agit de développer le besoin chez l'élève de situer les tableaux traités par rapport aux univers de référence à partir desquels ceux-ci ont pu prendre leurs sens. Il est important de clarifier que l'enseignement artistique à l'école n'est pas destiné à créer des artistes et que les cours d'arts visuels ne doivent pas être confondus avec des moments de pur plaisir synergétique. L'enseignement artistique doit être vu comme n'importe quelle autre discipline scolaire. Ainsi, tout cours d'arts visuels devrait être en rapport avec des contenus d'apprentissage, des objectifs et des compétences à faire acquérir aux élèves. Si dans la pratique l'accent est mis sur les capacités créatives des élèves cela ne veut pas dire récréation, loisirs et laisser-faire, mais la recherche d'une adéquation des activités aux enfants dans une tentative d'assurer la communication et l'apprentissage. D'autre part, il faut être clair sur le fait que cette méthode ne soutient pas le technicisme mais donne la priorité à la mesure des capacités transférées et transférables. Il ne suffit pas, en effet, de proposer de la peinture, des crayons ou des ciseaux aux élèves et de leur montrer des images pour pratiquer les arts visuels. Aujourd'hui, l'idée est de faire progresser la discipline par la recherche et d'obtenir en conséquence une meilleure utilisation de nouveaux concepts en sciences de l'éducation, psychologie, linguistique et sémiotique (Rickenmann, 2001). Sur la base de cette méthode, qui régit l'ensemble de mes activités, j'ai élaboré un projet d'apprentissage artistique basé sur la collection René et Madeleine Junod du Musée des Beaux Arts de la ville de La Chaux-de-Fonds. La démarche préalable fut pour moi d'étudier le contenu de la collection par le biais d'un catalogue, suivi de deux visites au musée pour analyser les propriétés et la conception de l'espace d'exposition et la distribution des œuvres. Quelle disposition physique ? Quels tableaux retenir pour les activités ? Le projet a ensuite été prévu en trois parties. La première partie a été réalisée en salle de classe avant la visite du musée. Elle a consisté en une activité de peinture fondée sur une interprétation libre de l'ekphrasis, c'est-à-dire basée sur des descriptions par des mots de certains tableaux de la collection et suivie de la « copie » de ces mêmes œuvres par la méthode de la grille de copie. La deuxième partie a consisté à réaliser, pendant la visite au musée, une série de «jeux de stimulation de l'observation ». Cette approche a renforcé et complété les activités de dessin et coloriage à la maison et en salle de classe pendant la troisième partie. Ces trois approches différentes ont donné aux élèves la possibilité de connaitre, de comprendre et d'apprécier l'existence de certains tableaux préalablement choisis et de réaliser leurs propres productions. Pour des raisons matérielles et physiques (place disponible, matériaux et outils spécifiques, possibilités d'aboutir à des réalisations gratifiantes, etc.), les choix techniques des activités 
pratiques ont été limités, l'un des critères de choix étant leur faisabilité au musée ainsi qu'en salle de classe.

\section{Les mots comme première rencontre de l'image}

L'enseignement artistique en arts visuels est généralement fondé sur l'observation d'œuvres d'art ou de reproductions sous forme de posters ou de diapositives, c'est-à-dire une introduction aux images par l'image. À l'inverse, pour leur première rencontre avec la collection René et Madeleine Junod, les élèves ont été confrontés à des mots qui décrivaient quatre tableaux différents, un exercice inspiré de l'ekphrasis littéraire. L'ekphrasis est un terme utilisé en linguistique pour définir une description orale ou écrite d'une œuvre avec l'intention d'engendrer des images mentales dans la tête de l'auditeur ou du lecteur. Il s'agit d'un exercice d'évocations d'images pour commencer à parler d'images. L'idée d'une étroite analogie entre la peinture et la pensée poétique permet de représenter la création artistique dans des termes qui conviennent aussi bien à la littérature qu'à l'analyse des tableaux. Si l'on part du principe qu'un tableau reproduit la pensée intime de l'artiste et que la nature est un dictionnaire de verbes et d'images où il va trouver ses sujets, les productions picturales, même figuratives, loin d'être une réponse simplement mimétique, susciteraient l'imagination du spectateur. Assimiler la peinture à des comptes rendus littéraires donnerait la possibilité d'agir de façon inverse : partir du texte pour récréer l'œuvre. Est-ce que les couleurs répondent aux paroles? Il a été proposé la description de quatre tableaux de styles, de genres et d'artistes différents. L'élève, selon la description de tableau choisie, a traduit en image ce qu'il a lu et s'est mesuré à diverses manières de traiter l'espace d'une feuille rectangulaire de papier en format A3. Chaque descriptif a posé des contraintes d'orientation du cadre, de centralisation de l'image, de verticalité ou d'horizontalité, de bas et de haut, de la ligne de base et de la ligne de sol pour placer les éléments décrits. Bref, chaque descriptif de tableau contient dans sa composition des notions concernant les composantes plastiques et leurs modes d'organisation (Reyt).

\section{Les tableaux retenus : idée d'exemple ou « variation dans le même »}

Les quatre tableaux choisis pour cette première activité font partie d'un ensemble qui concerne tout le projet. Pour les choisir, il a fallu s'appuyer d'abord sur deux questions : Qu'est-ce qu'une collection d'œuvres d'arts ? Pourquoi constituer une collection ? Pour y répondre, nous avons dégagé deux fonctions essentielles d'une collection : la beauté et la singularité. Or, les tableaux de la collection Junod constituent, par leur diversification, un bon 
exemple d'une collection efficace (Charrière). L'ensemble des paramètres artistiques mis en rapport dans un tableau (lignes, couleurs, masses, lumières...) a conduit, en histoire de l'art, à ce que, selon le genre, l'artiste ou l'époque, certains soient davantage pris en considération que d'autres. Pour élargir les rencontres entre les œuvres et les élèves, ont été pris comme critères :

- Les différences entre certains genres picturaux ;

- Les différents modèles spatiaux ;

- L'organisation et la richesse picturale de chaque tableau.

La collection Junod étant composée exclusivement de tableaux « figuratifs », les descriptions concernent l'agencement des éléments du tableau dans un souci d'organisation séquentielle.

\section{La constitution d'un vocabulaire}

S'il s'agissait, pour simplifier, d'apprendre un vocabulaire par le biais d'actions plastiques sur un support ordinaire (papier), il allait de soi que ces actions plastiques nécessitaient une compréhension de l'abécédaire basique des arts visuels. Donc, cette première activité a consisté en un va-et-vient entre mots et images dans une totale réciprocité. Avant de représenter plastiquement les descriptions, les élèves ont tous lu les quatre textes présentant les quatre tableaux retenus et les ont commentés et questionnés oralement. Cependant, si l'objectif initial était l'acquisition, par ces activités, d'un vocabulaire artistique commun et d'un savoir-faire technique, d'autres objectifs se sont dégagés pendant la réalisation : avoir une réflexion spécifique pour chaque description, découvrir des façons de procéder différentes, questionner et comparer, sans jugement de valeurs, des points de vue. En référence à Claude Reyt, l'accent a été placé sur les notions :

- d'utilisation de l'espace sur une surface ;

- de types de compositions: disposition des parties dans l'œuvre, distribution des lumières ;

- de plans d'observation : $1^{\mathrm{e}}$ plan, $2^{\mathrm{e}}$ plan ;

- d'échelles de plans (notions topologiques) : près/loin, devant/derrière ;

- de volumes : vide/plein, fond/forme ;

- d'utilisation des couleurs : façon symbolique, caractéristiques des touches, idée de contraste...

Il a été intéressant de constater déjà, lors d'une première lecture des quatre récits, comment et pourquoi les multiples façons de traiter l'espace dépendent de plusieurs paramètres artistiques 
et particulièrement du genre du tableau, selon qu'il s'agit d'un portrait, d'un paysage, d'une nature morte ou encore d'un récit historique. Il a fallu aussi, dans un premier temps, aiguiller les élèves afin qu'ils prennent conscience de la place des différents éléments dans les compositions. Par exemple : premier plan, deuxième plan, gauche, droite, etc. Après la lecture collective de chaque description nous avons entrepris la partie pratique. D'abord les élèves ont commencé à produire leur dessin au crayon de papier et à la gomme en suivant les descriptions proposées. L'étape du crayon permet davantage de détails et les élèves ont d'ailleurs l'habitude de l'utiliser à cette fin. La seconde séquence était réservée à la peinture et aux consignes de couleurs énoncées par la description.

\section{Les descriptions et les tableaux}

Ci-dessous l'exemple de deux des quatre tableaux choisis, basés sur les écrits du catalogue Collection René et Madeleine Junod (1993).

Chaque présentation commence par une image du tableau original accompagnée d'un résultat de l'exercice d'ekphrasis réalisé par un élève en suivant lesdites descriptions.

« La fuite en Égypte » (1942) - Georges Rouault

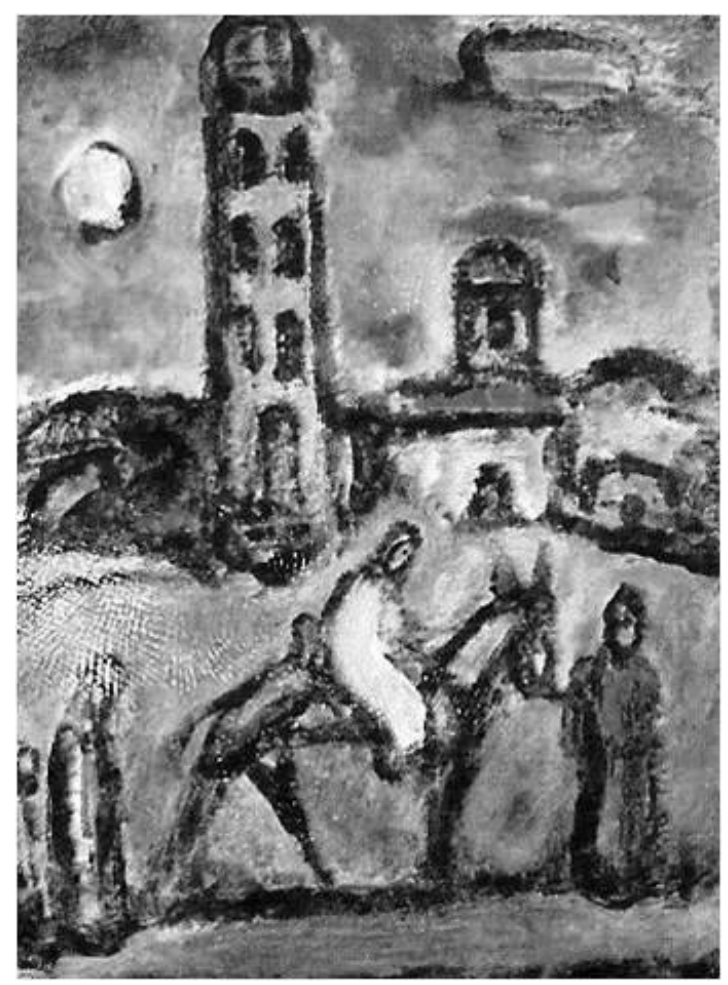




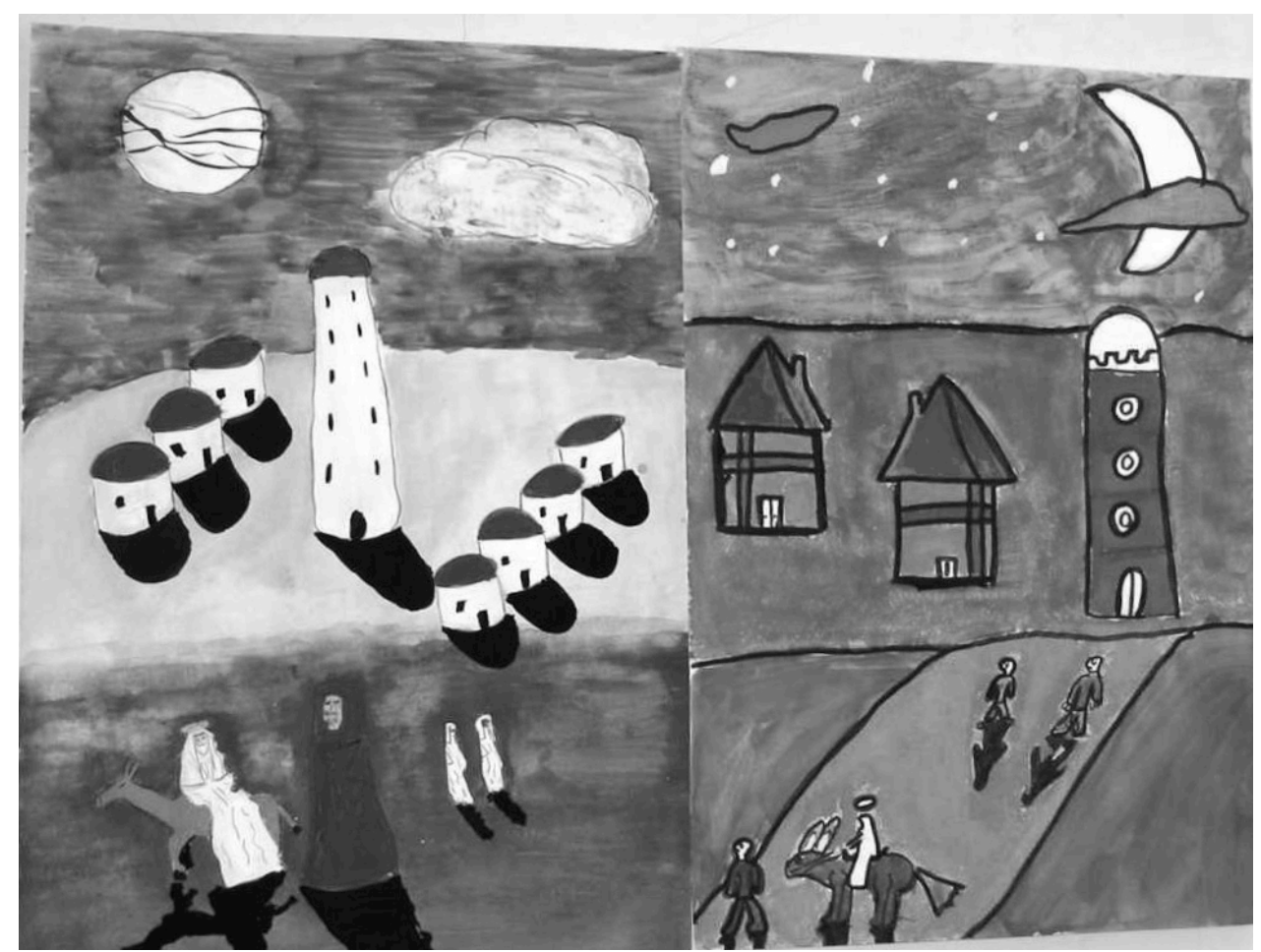

Au troisième plan on voit un ciel bleu crépusculaire avec des nuages et une lune blanche. Au deuxième plan des maisons, une tour et une prairie. Au premier plan un sol argileux avec cinq personnages: quatre humains et un âne. Un des personnages est sur l'âne. Les ombres des personnages sont reflétées sur le sol en bas du tableau. Elles sont reflétées comme si la lumière venait du zénith. L'espace suggéré à grands traits est dominé par une tour ou un minaret à coupole, symbolisant le temple. On peut voir de chaque côté de cette tour des maisons et une prairie lointaine. On y trouve trois personnages bien définis au premier plan: Marie, l'âne et Joseph. Deux autres personnages sont esquissés, mais sont difficiles à définir : un enfant, une femme, un homme? Ils marchent derrière le trio. La tête de Marie, auréolée de lumière, occupe le centre de la composition dominée par l'impressionnante tour qui relie la terre au ciel. Son vêtement est de la même blancheur laiteuse que la lune dans le ciel bleu au troisième plan. Le rouge du manteau de Joseph est le même que sur les toits des maisons au deuxième plan. Le tableau est coloré comme un vitrail. Le pourtour des éléments (architecture, personnages prairie, lune) est épaissi par un trait de couleur noire.

En choisissant cette description l'élève a été confronté, plus spécifiquement, aux notions de traits (types de tracés), de plans et de distributions harmonieuses de couleurs. Le tracé vient déterminer le geste et la composition des éléments. Et c'est par l'aménagement des différents plans proposés par la description que l'élève arrivera à l'impression de profondeur. Pour cela il doit connaitre la distinction, dans un schéma traditionnel, entre les trois catégories suivantes : premiers plans, plans intermédiaires, lointains (Reyt). Quant aux couleurs, elles se présentent par répétitions dans un jeu de contrastes et deviennent indissociables des rendus de lumière et de l'idée de vitrail. Parmi d'autres connaissances engagées dans cette description, 
on retrouve une approche religieuse (narratif biblique) et la compréhension des représentations des vitraux.

« La fillette aux cheveux ébouriffés » (1888) - Vincent Van Gogh

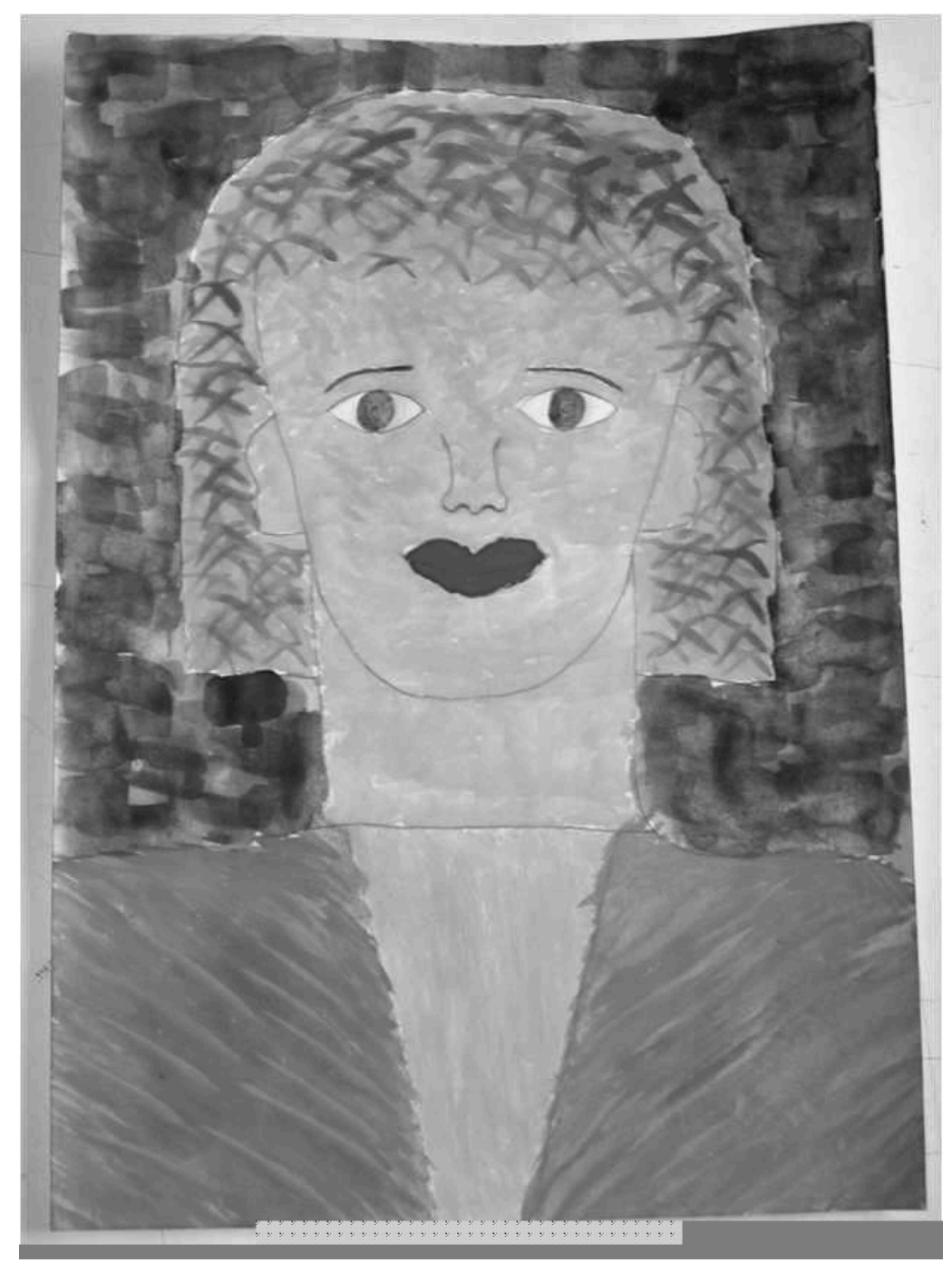




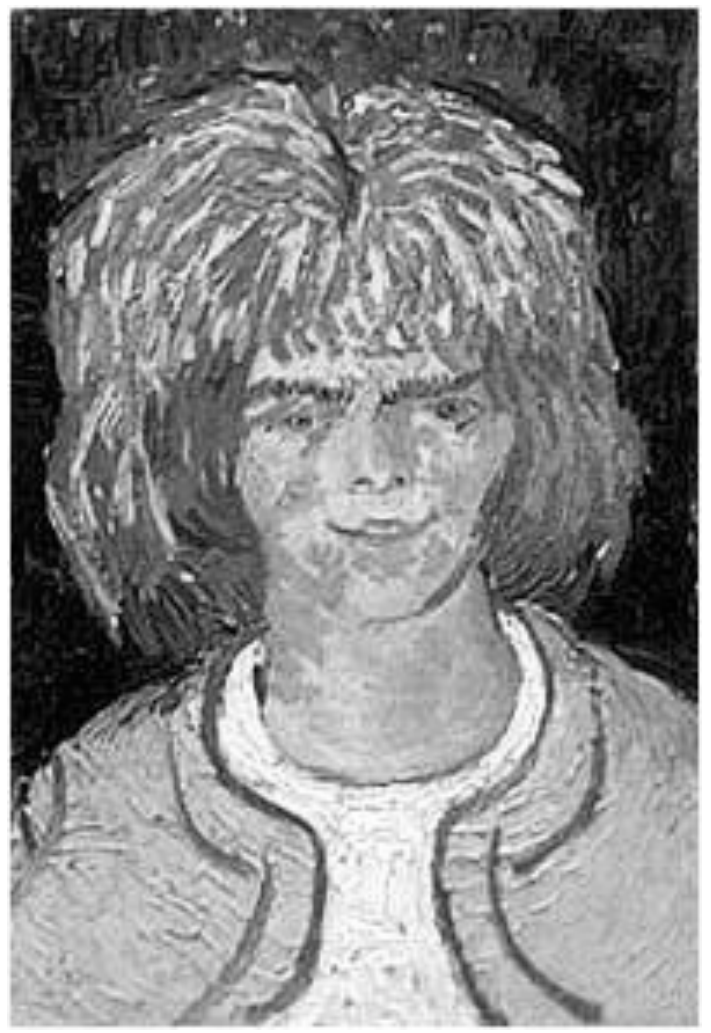

Sur un fond bleu foncé on voit le portrait d'une fillette représenté en buste et frontalement. Son visage est gai. Elle sourit. Ses yeux sont verts et la couleur de sa peau est jaunatre. Elle porte une chevelure flamboyante dans les tons d'orange et jaune. Son costume est simple : un T-shirt jaune avec une jaquette bleu clair. Le portrait qui occupe toute la surface du tableau est peint par des contrastes de tons purs. Il y a une organisation symétrique de touches colorées en zones délimitées chacune par un système de signes : touches horizontales pour le fond, striées pour le visage, courbes et entrecroisées pour la chevelure, verticale pour le T-shirt et obliques pour la jaquette.

Cette description d'un portrait individuel contient des indications rigides à la fois de cadrage (le portrait en buste et de face) et des indications sur le modèle (sexe et âge). Ces caractéristiques déterminent le dessin: le portrait frontal d'une fillette. Les élèves connaissaient déjà les proportions d'un visage et un peu l'œuvre de Van Gogh. Son style unique, avec des touches de peinture épaisses, larges et hachurées, faisait partie de leurs connaissances en histoire de l'art et ont été rapidement investies. L'idée principale était de prendre conscience de l'importance de varier, par les touches, le rythme du tableau. D'une manière plus précise, dans l'œuvre de Van Gogh, il était essentiel de comprendre cette relation entre les manipulations effectuées par les couleurs pures et les touches (horizontales, verticales, courbes) et l'obtention de certains effets visuels et symboliques concernant la représentation d'un visage. 


\section{Les limites concernant l'activité de « décrire une image par des mots »}

Afin de développer l'esprit critique et d'analyse chez les élèves, que ce soit dans les séquences d'enseignement de l'observation d'œuvres ou pendant leurs productions, il a fallu créer des repères communs par le biais d'un vocabulaire spécifique aux arts visuels. Une fois l'activité libre de l'ekphrasis mise en place, les élèves ont échangé leurs observations et ont travaillé les couleurs et les mélanges approximatifs en investissant des connaissances pratiques déjà utilisées auparavant. Cette activité a été source de dialogues entre les élèves, chacun essayant de convaincre l'autre de sa perception et précisant ses arguments au fur et à mesure de sa réalisation plastique. Pour stimuler la recherche de solutions et favoriser les échanges verbaux, les élèves ont pu débattre en dyade pendant toute la partie de production. Il a été très intéressant de les rapprocher par leur choix de descriptions et de les voir chercher dans leurs productions les ressemblances et les différences. Même en travaillant côte à côte, les élèves ont fait preuve d'originalité, transférant et réinvestissant leurs connaissances discutées en groupe. L'utilisation par tous les élèves d'un vocabulaire approprié à la discipline a été demandée tout au long de l'activité. Mais la compréhension individuelle de chaque description a aussi fait écho à leurs expériences antérieures, à ce qu'ils connaissaient déjà et à leur degré de familiarité avec les techniques. Comme mentionné auparavant, dans la continuité de cette activité et pour réaliser leur nouvelle production par la grille de copie, les élèves ont dû recourir à une pratique d'observation sur une image plane, à partir de la photocopie du tableau choisi auparavant. Cette activité facilite un contrôle des résultats obtenus à chaque étape du dessin par une observation très précise du modèle. Mais ce contrôle doit être fait avec le plus grand soin et avec la plus grande précision dans les traits car, en suivant le système de grille numérotée, le dessin de chaque carreau doit être parfaitement réalisé afin de permettre l'existence en continuité des autres. Enfin, en conclusion de l'exercice, ils ont photographié leurs productions et nous les avons exposées en classe. Cette présentation des œuvres au travers de textes et d'images a contribué à renforcer et orienter le désir des élèves d'aller voir les œuvres authentiques. Après cette approche indirecte de la collection est venu le moment d'un rapport direct à la réception des œuvres au musée. Cette expérience physique en face des tableaux avait pour objectif d'ancrer les peintures de la collection dans l'imaginaire des élèves, dans leurs expériences et leurs mémoires personnelles. Ce face à face avec les œuvres est considéré par la Proposition triangulaire comme la condition de l'appropriation par les élèves des expressions plastiques des artistes et de la valorisation d'une culture commune (Barbosa). 


\section{Réflexions}

Pendant le déroulement de l'activité mentionnée dans cette contribution, certains contenus plastiques et formels des œuvres ont été travaillés. Les descriptifs proposés ciblent des composants comme la couleur, les modes d'organisation et les modes de relations entre les éléments d'un tableau. Le choix des œuvres a été directement lié aux concepts et notions à étudier. Ce type d'exercice permet de travailler ces contenus et composantes plastiques de façon, parfois, très directive. La réduction à un seul tableau et à une seule description détermine le cheminement de la production de l'élève. Cette réflexion doit être prise en compte dans la progression des activités du projet afin de permettre la compréhension des résultats de chaque étape et le prolongement, réinvestissement, des capacités acquises dans les exercices suivants. Concernant les descriptions proposées, l'activité de l'ekphrasis voulait mobiliser, dans la tradition occidentale, le genre textuel spécifique qui s'est principalement développé dans le sillage de l'histoire de l'Art descriptive et de la critique d'art. Du fait de l'absence d'une référence explicite à ces genres textuels, l'utilisation des descriptions, a été naturalisée dans cette séquence. Autrement dit, elle suppose que chacun peut décrire une œuvre d'art de la même manière et à sa manière, ce qu'historiquement et textuellement est loin d'être le cas. Il serait donc très intéressant d'explorer les différents niveaux lexicaux, textuels et discursifs utilisés pour décrire et analyser une œuvre picturale par les critiques et historiens d'art. Quels effets une telle démarche aurait sur la construction d'une conception critique des œuvres, non seulement de celle étudiée mais aussi des tableaux de référence ? Ce type d'activité pourrait être réalisé en référence aux grands classiques du genre littéraire de critique d'art comme les comptes rendus des Salons par Baudelaire, les écrits de Valéry ou de Starobinski. Par exemple, en parlant de peinture, Baudelaire a voulu réfléchir sur l'esthétique, c'est-à-dire sur les conditions d'émergence d'une beauté et des façons de la mettre en mots et en images. Souligner le potentiel d'une telle démarche pourrait, pour autant qu'elle soit prise en charge par l'enseignant comme un des objets d'enseignement de l'activité, enrichir les productions plastiques. Toutes ces remarques sont importantes pour comprendre les dynamiques qui se créent dans les activités qui cherchent la mobilisation des connaissances acquises dans des disciplines différentes, ici la lecture, la compréhension de texte et la réalisation d'une production artistique. C'est pourquoi il parait important de poursuivre l'exploration de ce type d'activité. Cerner plus précisément les contenus, les prérequis, mais aussi les marges de manœuvre des élèves constitue un vaste champ d'étude qui mérite d'être exploré, tout particulièrement dans le contexte actuel où se développe l'importance de l'interdisciplinarité. 


\section{Bibliographie}

Barbosa, Ana-Mae. A image no ensino da arte. Guarulhos : Perspectives, 1991.

Charrière, Edmond. Collection René et Madeleine Junod. La Chaux-de-Fonds : Musée des Beaux-Arts, La Chaux-de-Fonds, 1993.

Reyt, Claude. Enseigner les arts visuels. L'image au cycle 3. Paris : Bordas, 2005.

Rickenmann, René. Les arts plastiques à l'école primaire. CD-Rom, Cahiers de la Section des sciences de l'éducation 96. Genève : U de Genève FPSE, 2001. 